\title{
COPD: Eine entzündliche Erkrankung der Atemwege?
}

\section{H. Magnussen}

\author{
COPD: An Inflammatory Disease of the Airways?
}

\section{Einleitung}

Die chronisch obstruktive Bronchitis und das Lungenemphysem werden aufgrund der Schwierigkeiten der Abgrenzung der bronchitischen und emphysematösen Komponente nach dem Vorschlag der Global Initiative for Chronic Obstructive Lung Disease (GOLD) mit COPD (chronic obstructive pulmonary disease) abgekürzt [1].

Die COPD ist durch eine nicht vollständig reversible Begrenzung des Atemflusses charakterisiert. Die Limitation des Atemflusses ist meist fortschreitend und mit einer abnormen entzündlichen Reaktion der Lungen vergesellschaftet [1,2].

Die Pharmakotherapie der COPD beruht überwiegend auf den Substanzen, die bei der Behandlung des Asthma bronchiale eingesetzt werden. Die Atemwegsobstruktion kann erfolgreich mit Bronchodilatatoren behandelt werden, während für die Beeinflussung der abnormen entzündlichen Reaktion bislang vorwiegend Kortikosteroide zur Verfügung stehen. Die hervorragende Effektivität der Kortikosteroide beim Asthma bronchiale hat zu zahlreichen Studien geführt, die den Einsatz insbesondere von inhalativen Kortikosteroiden bei der COPD rechtfertigen sollen.

Ich möchte mich in dem folgenden Beitrag mit einigen Aspekten auseinandersetzen, die den Zusammenhang zwischen den funk- tionellen Konsequenzen der COPD, der abnormen Entzündungsreaktion und den therapeutischen Möglichkeiten betreffen.

\section{Funktionelle Konsequenzen der COPD}

Die COPD ist durch eine meist progressive Limitation der Strömung innerhalb der Atemwege gekennzeichnet. Dieses funktionelle Merkmal wird üblicherweise mit Hilfe der Spirometrie gemessen, die aufgrund der internationalen Übereinkommen nur das exspiratorische Manöver berücksichtigt [3]. Die forciert exspirierte Vitalkapazität, FVC, das forciert exspirierte Volumen in der ersten Sekunde der Ausatmung, $\mathrm{FEV}_{1.0}$ und deren Verhältnis beschreiben die Atemwegsobstruktion in qualitativer und quantitativer Weise. Die Stadieneinteilung der COPD beruht auf dem Nachweis einer obstruktiven Ventilationsstörung durch eine Erniedrigung von $\mathrm{FEV}_{1.0} / \mathrm{FVC}<70 \%$ sowie einer definierten Erniedrigung von $\mathrm{FEV}_{1.0}$. Während die spirometrischen Parameter von den verschiedenen Expertengremien in gleicher Weise eingesetzt werden, unterscheiden sich die quantitativen Angaben, die zur Abschätzung der Schweregrade führen. Dieser Umstand erschwert unter anderem den Vergleich epidemiologischer Erhebungen zur Häufigkeit und Schwere der COPD in verschiedenen Ländern $[4,5]$.

Die Objektivierung der obstruktiven Ventilationsstörung durch die alleinige Auswertung der forcierten Exspiration stellt einen

Institutsangaben

Krankenhaus Großhansdorf, Zentrum für Pneumologie und Thoraxchirurgie

Widmung

Diese Arbeit ist meinem Kollegen Detlef Kirsten gewidmet, mit dem ich im gleichen Jahr 60 Jahre alt werde. Die Loyalität und Freundschaft, die uns verbindet, hat auch unser Krankenhaus Großhansdorf geprägt. Die Mitarbeiter und ich gratulieren und danken ihm. Ich freue mich auf weitere Jahre der beruflichen und persönlichen Begegnung.

Korrespondenzadresse

Prof. Dr. Helgo Magnussen · Krankenhaus Großhansdorf · Zentrum für Pneumologie und Thoraxchirurgie · Wöhrendamm 80·22927 Großhansdorf·E-mail: magnussen@pulmoresearch.de

Eingang: 18. Februar 2004 · Angenommen: 20. Februar 2004

Bibliografie

Pneumologie 2004; 58: 320-324 • @ Georg Thieme Verlag KG Stuttgart · New York

DOI 10.1055/s-2004-818401

ISSN 0934-8387 
Kompromiss dar, der der Überlegung folgt, dass eine Messung eines Aspektes der Lungenfunktion besser ist, als auf jegliche Objektivierung zu verzichten. Daher werden einfache, robuste und preiswerte Spirometer empfohlen, die jedoch den Nachteil haben, die Inspiration nicht zu erfassen [6].

$\mathrm{FEV}_{1.0}$ beschreibt den Schweregrad der COPD und das bereits historische Werk von Fletcher, Peto, Tinker und Speizer [7] beschreibt den Zusammenhang zwischen $\mathrm{FEV}_{1.0}$ und Prognose der Erkrankung. In jüngster Zeit konnte allerdings gezeigt werden, dass die Mortalität der COPD besser durch das Ausmaß der Einschränkung der 6-Minutengehstrecke beschrieben wird, als durch $\mathrm{FEV}_{1.0}[8,9]$.

Die Therapie mit Bronchodilatatoren bei der COPD führt häufig nur zu einer geringen Zunahme von $\mathrm{FEV}_{1.0}$, obwohl die Patienten eine subjektiv empfundene Besserung angeben. Da die Patienten ihre Atembeschwerden als Behinderung der Einatmung beschreiben und die erfolgreiche Therapie als Verbesserung der Einatmung artikuliert wird (language of dyspnoe in COPD), ist der Bedeutung der inspiratorischer Parameter der Lungenfunktion zunehmende Bedeutung geschenkt worden. O'Donnell hat in mehreren Arbeiten das Konzept der dynamischen Lungenüberblähung bei körperlicher Belastung durch experimentelle Befunde gestützt $[10-12,8]$. Die Überblähung der Lunge, die im deutschsprachigen Raum meist durch eine Vergrößerung des ganzkörperplethysmographisch gemessenen intrathorakalen Gasvolumens gemessen wird, geht mit einer Einschränkung der inspiratorischen Kapazität, IC, einher. Aufgrund der weitgehenden Konstanz der totalen Lungenkapazität, TLC, ist daher in Ländern, die kaum über Ganzkörperplethysmographen in der klinischen Routine verfügen, die Messung der IC zur Objektivierung der Überblähung empfohlen worden. Wir haben kürzlich in Zusammenarbeit mit O'Donnell zeigen können, dass das langwirksame Anticholinergikum Tiotropium die dynamische Lungenüberblähung bei körperlicher Belastung vermindert und gleichzeitig die körperliche Belastbarkeit verbessert. Die Korrelation zwischen der Zunahme der körperlichen Belastung sowie den spirometrischen und ganzkörperplethysmographischen Parametern der Lungenüberblähung war wesentlich enger als die entsprechenden Korrelationen mit $\mathrm{FEV}_{1.0}$ [13]. Diese Arbeit belegt erneut, dass der Nutzen einer therapeutischen Intervention bei der COPD nur unvollkommen mit $\mathrm{FEV}_{1.0}$ beschrieben werden kann. Wir hatten überdies kürzlich beschrieben, dass der bronchodilatorische Effekt eines $\beta_{2}$-Sympathikomimetikums besser durch das forciert eingeatmete Volumen in der ersten Sekunde der Einatmung, FIV $_{1.0}$ als durch sein Analogon der Ausatmung $\mathrm{FEV}_{1.0}$, charakterisiert werden kann [14]. Die Dissoziation von $\mathrm{FEV}_{1.0}$ und FIV ${ }_{1.0}$ in Bezug auf die Änderung der Dyspnoe gilt nur für Patienten mit mittelschwerer bis schwerer COPD und nicht für Patienten mit mittelgradig schwerem Asthma bronchiale [15].

Die funktionellen Konsequenzen, die den pathologisch-anatomischen Veränderungen der COPD folgen, lassen sich daher nur unvollkommen mit dem Parameter $\mathrm{FEV}_{1.0}$ beschreiben. Die Determinanten von $\mathrm{FEV}_{1.0}$ sind komplex und werden von der Integrität der Atemwege, der Interaktion zwischen Atemwegen und Lungenparenchym sowie dem Lungenvolumen bestimmt.
Die neuen Erkenntnisse über die Bedeutung der inspiratorischen Limitation der Atmung, der Lungenüberblähung und der eingeschränkten körperlichen Belastbarkeit sind bisher nur unvollkommen in die Interpretation morphologischer Befunde und die Beurteilung therapeutischer Maßnahmen eingegangen.

\section{Abnorme Entzuindlungsreaktion}

\section{Mikroskopische Befunde}

Der Zusammenhang zwischen zellulären, strukturellen und funktionellen Veränderungen bei der COPD ist besonders von der Arbeitsgruppe um M. Saetta beschrieben worden. In ihrem kritischen Übersichtsartikel werden folgende Schlussfolgerungen hervorgehoben: (a) alle Raucher entwickeln eine Entzündung der Atemwege (b) die Entzündungsreaktion wird insbesondere während einer Exazerbation verstärkt (c) die inflammatorische Reaktion korreliert mit der Schwere der Erkrankung (d) die Inflammation ist auch nach Beendigung des Rauchens nachweisbar (e) alle Entzündungszellen sind an dem inflammatorischen Prozess beteiligt (f) eine Vielzahl von Mediatoren werden von den aktvierten Entzündungszellen freigesetzt (g) der oxidative Stress, der mit dem Rauchen verbunden ist, trägt zur Pathogenese der COPD bei. Trotz der Klarheit dieser Aussagen ist die Pathogenese der COPD in weiten Teilen unverstanden [16]. Die Arbeitsgruppe konnte weiterhin zeigen, dass bei dem Vergleich der kleinen Atemwege von Rauchern mit schwerer und leichter COPD die schwere COPD eine erhöhte Zahl von Leukozyten aufweist, die mit der der Einschränkung von $\mathrm{FEV}_{1.0}$, der Lungenüberblähung, der erniedrigten Dco sowie dem radiologischen Ausmaß des Lungenemphysem korrelierten. Der inflammatorische Prozess war im Wesentlichen durch eine vermehrte Zahl von T-Lymphozyten und Makrophagen charakterisiert [17]. Diese Befunde sind im Einklang mit den Daten aus der Gruppe um J. Hogg, der überdies zeigen konnte, dass das schwere Lungenemphysem mit einer vielfachen Erhöhung der Expression des adenoviral transactivating protein, E1A, in alveolaren Epithelzellen einhergeht [18]. Die Vielzahl neuer Erkenntnisse lässt aber weiterhin Fragen zur Pathogenese der COPD offen, so dass kürzlich die Hypothese aufgestellt wurde, ob die COPD ein autoimmunologisches Geschehen aufweist [19]. In unserer Arbeitsgruppe werden die Wachstumseigenschaften isolierter Lungenfibroblasten von Patienten mit Emphysem in Kultur untersucht. Diese Zellen haben eine bedeutsame Funktion in der Integrität des Lungenparenchyms. Die Beobachtung, dass diese Zellen im Vergleich zu Fibroblasten aus dem Parenchym von Patienten ohne COPD langsamer wachsen, spricht für die Hypothese, dass die Lungenfibroblasten bei der Entstehung des Emphysems eine zentrale Rolle spielen [20].

\section{Nicht invasive Untersuchungen der Atemwegsentzündung}

Die bislang geschilderten Befunde beruhen auf Untersuchungen von bioptisch oder chirurgisch entnommenen Lungengewebe. In den vergangenen Jahren sind aber zahlreiche Methoden zur Objektivierung der Atemwegsentzündung mit Hilfe nicht - invasiver Methoden entwickelt worden, die die Untersuchung der Atemwegsentzündung in wesentlich größeren Gruppen, bei gleichzeitig deutlich minimiertem Risiko erlauben. 
Zur Erfassung entzündlicher Vorgänge in den Atemwegen bei der COPD ist im Wesentlichen die Sputuminduktion geeignet. Die Messung von Stickstoffmonoxid in der ausgeatmeten Luft ist bei der COPD bislang nur begrenzt sinnvoll, insbesondere solange die Patienten noch rauchen. Die Bewertung des Atemkondensates zur Messung der Atemwegsentzündung bei der COPD. ist noch nicht abschließend möglich.

\section{Induziertes Sputum}

Die Methodik der Sputuminduktion bei COPD ist etabliert [21,22]. Es sollte jedoch berücksichtigt werden, dass die Sputuminduktion mit hypertoner Kochsalzlösung bei der Mehrzahl der Patienten mit COPD eine klinisch bedeutsame obstruktive Ventilationsstörung hervorruft, die auch durch eine vorherige Inhalation eines $\beta_{2}$-Agonisten nicht verhindert werden kann [23]. Da Patienten mit COPD in der Regel Sputum auch spontan produzieren können, reicht meist die Induktion mit isotoner Kochsalzlösung aus, um Sputum in ausreichender Menge zu erhalten. Dennoch ist im klinischen Alltag die Sputuminduktion nur unter geeigneten Vorsichtsmaßnahmen zu empfehlen. Dieser Umstand, sowie die relativ zeitaufwendige Aufbereitung und Analyse des induzierten Sputums stehen einer größeren Verbreitung dieser Technik im klinischen Alltag entgegen.

Die zelluläre Zusammensetzung des induzierten Sputums zeigt Unterschiede zwischen Asthma und COPD $[24,22,25]$. Im stabilen Krankheitszustand überwiegen bei der COPD die neutrophilen Granulozyten. Da der Anteil dieser Zelltypen im Sputum aber sehr variabel ist, lassen sich selten diagnostische Rückschlüsse ziehen. Eine gleichzeitig deutlich erhöhte Gesamtzellzahl/mL Sputum deutet auf eine infektive Bronchitis hin [26]. Eine Subpopulation von Patienten mit COPD kann jedoch auch vermehrt eosinophile Granulozyten im Sputum aufweisen [27]. Dieser Befund ist möglicherweise von Bedeutung, da er die therapeutische Effektivität von inhalativen Kortikosteroiden bei der COPD vorhersagen lässt [27]. Eosinophile Granulozyten im Sputum nehmen auch während einer Exazerbation der COPD zu.

Im Sputumüberstand können auch Entzündungsmediatoren bestimmt werden, die Rückschlüsse auf die Aktivierung der Zellen zulassen. So gehen beispielsweise erhöhte Konzentrationen von IL-8 und MPO mit erhöhten Anteilen von neutrophilen Granulozyten einher. Die Messung von Mediatoren im Sputumüberstand erfordert aber in der Regel eine recht aufwendige Aufarbeitung, und viele Mediatoren werden durch die Homogenisation des Sputums beeinflusst [28].

\section{Stickstoffmonoxid in der Ausatemluft}

Stickstoffmonoxid, NO, ist ein Gas, welches mit Hilfe teurer, kommerziell erhältlicher Messgeräte schnell und reproduzierbar in der Ausatemluft gemessen werden kann. Die Messung von NO in der Ausatemluft ist nicht-invasiv und leicht durchführbar, so dass diese Methode auch eine hohe Attraktivität für die pädiatrische Pneumologie besitzt. Es besteht eine relativ enge Korrelation zwischen der ausgeatmeten NO-Konzentration und dem Anteil der eosinophilen Granulozyten des Atemtraktes. Daher könnte die
Methode bei der COPD evtl. auch zur Identifikation von Patienten dienen, die auf eine Steroidtherapie ansprechen [27]. Bei Patienten mit COPD, die weiterhin rauchen, muss berücksichtigt werden, dass Zigarettenrauch durch seine hohe NO-Konzentration zur Abregulation der NO-Produktion in den Atemwegen führt [29].

\section{Atemkondensat}

Die Sammlung von Atemkondensat ist einfach und mit kostengünstigen Apparaturen durchführbar. Kommerzielle Geräte stehen für eine standardisierte Gewinnung zur Verfügung. Die Methode ist für den Patienten leicht erlernbar, da keine bestimmten Atemmanöver erforderlich sind. Die Lagerung und Analyse des gewonnenen Materials sind wesentlich komplizierter und abhängig vom Parameter, der gemessen werden soll. Der Aufwand und die Kosten sind denen der Messung eines Sputumüberstandes vergleichbar.

Bei der COPD wurden bereits zahlreiche Parameter erhöht im Atemkondensat gefunden. Dies gilt insbesondere für die Exazerbation der Erkrankung. $\mathrm{H}_{2} \mathrm{O}_{2}$ wird z. B. als ein Marker des oxidativen Stresses angesehen und könnte bei der Pathogenese der COPD einen wesentlichen Faktor darstellen [30]. $\mathrm{H}_{2} \mathrm{O}_{2}$, Isoprostane und andere Parameter der Entzündung im Atemkondensat sind allerdings auch beim Asthma oder der zystischen Fibrose erhöht gefunden worden, und somit ist eine solche Messung für die Diagnostik wenig geeignet [31].

Die Messgenauigkeit und Reproduzierbarkeit der $\mathrm{H}_{2} \mathrm{O}_{2}$-Messung sind im Allgemeinen schlechter als bei der Bestimmung von NO. Wir haben auf die Schwierigkeit der Reproduzierbarkeit von $\mathrm{H}_{2} \mathrm{O}_{2}$ im Exhalat hingewiesen [32]. Dieser Befund wurde jüngst in eindrucksvoller Weise bestätigt und bezieht sich auch auf andere Parameter im Atemkondensat wie Isoprostan [33]. Aus diesen Gründen erscheint es auch z.Zt. wenig wahrscheinlich, dass sich die Messung des Atemkondensats im klinischen Alltag durchsetzen wird.

Zusammenfassend kann man feststellen, dass mit Hilfe unterschiedlicher Methoden eine abnorme Entzündungsreaktion bei der COPD festgestellt werden kann. Im Gegensatz zum Asthma bronchiale ist jedoch die Entzündungsreaktion weniger spezifisch und kann nur unvollkommen durch semi-invasive (induziertes Sputum) und nicht-invasive (Ausatemluft) Verfahren abgebildet werden.

\section{Antiinflammatorische Therapie der COPD}

Glukokortikosteroide stellen die wirksamste antiinflammatorische Substanzgruppe beim Asthma bronchiale dar. Die gelegentlichen Schwierigkeiten der differenzialdiagnostischen Abgrenzung von Asthma und COPD [34], der Nachweis einer abnormen Entzündungsreaktion bei der COPD, sowie das Fehlen eines wirksamen antiinflammatorischen Therapieprinzips bei der COPD haben dazu geführt, dass das Leiden der Patienten weiterhin mit Kortikosteroiden behandelt wird. 
Die systemische Kortikosteroidtherapie des Patienten mit stabiler COPD führt zu einer Verschlechterung des Krankheitsbildes [35]. Systemische Kortikosteroide sind daher nur für den begrenzten Zeitraum einer Exazerbation indiziert [36]. Die vierwöchige Behandlung von Patienten mit leichter bis mittelschwerer COPD mit hochdosierten inhalativen Kortikosteroiden führt weder zu einer funktionellen Verbesserung noch zu einer Modulation der Entzündungsmarker im Sputum und Exhalat [37,38]. Vor dem Hintergrund dieser Daten sollte der Einsatz von systemischen Kortikosteroiden der Exazerbation vorbehalten bleiben [39]. Die Empfehlung zur inhalativen Kortikosteroidtherapie der stabilen mittel bis schwergradigen COPD wird weiterhin diskutiert.

Große prospektive klinische Studien haben gezeigt, dass inhalative Kortikosteroide den Krankheitsverlauf der COPD nur wenig [40] oder nicht [41] verändern können. Die Exazerbationsrate verminderte sich jedoch im Verhältnis zu Ausmaß der eingeschränkten Lungenfunktion [42]. Retrospektive, epidemiologische Untersuchungen legten eine Verminderung der Mortalität bei Patienten nahe, die wegen einer Exazerbation stationär behandelt wurden [43]. Es wurde jedoch überzeugend belegt, dass diese erwartungsvolle Aussage auf einer fälschlichen Zuordnung der Dauer der inhalativen Steroidtherapie beruhte [44,45]. Die Kombination von lang wirksamen $\beta_{2}$-Agonisten mit inhalativen Kortikosteroiden hat in mehreren Studien zu einer Verbesserung der Lungenfunktion, Verminderung der Symptome sowie Abnahme der Exazerbation geführt [46]. Diese Effekte wurden bei der alleinigen Therapie mit lang wirksamen $\beta_{2}$-Agonisten nicht beobachtet [47].

Beim Asthma bronchiale wird bei nachgewiesener Reversibilität einer Atemwegsobstruktion ein günstiger Effekt der inhalativen Steroide vorausgesagt. Dieser Zusammenhang konnte jedoch für die COPD nicht gezeigt werden [48].

Der Nachweis der therapeutischen Überlegenheit einer Kombinationstherapie mit langwirksamen $\beta_{2}$-Agonisten und inhalativen Steroiden bei der mittelschweren und schweren COPD kann nicht einfach erklärt werden. Es ist wenig wahrscheinlich, dass der Effekt auf eine Beeinflussung eines inflammatorischen Prozesses zu beziehen ist, der für die COPD beschrieben wurde. Das lang wirksame Anticholinergikum Tiotropium besitzt keine antiinflammatorische Wirkung. Es konnte jedoch eine Verbesserung der Lungenfunktion, der Belastbarkeit und eine Abnahme der Exazerbation beschrieben werden [49].

Zukünftige Untersuchungen müssen helfen, unser Verständnis der komplexen Interaktion zwischen Symptomen, Funktion, zellulären Mechanismen sowie therapeutischen Effekten bei der COPD zu verbessern.

\section{Literatur}

${ }^{1}$ Pauwels RA, Buist AS, Calverley PM et al. Global strategy for the diagnosis, management, and prevention of chronic obstructive pulmonary disease. NHLBI/WHO Global Initiative for Chronic Obstructive Lung Disease (GOLD) Workshop summary. Am J Respir Crit Care Med 2001; 163: $1256-1276$
${ }^{2}$ Worth H, Buhl R, Cegla U et al. Leitlinie der Deutschen Atemwegsliga und der Deutschen Gesellschaft für Pneumologie zur Diagnostik und Therapie von Patienten mit chronisch obstruktiver Bronchitis und Lungenemphysem (COPD). Pneumologie 2002; 56: 704-738

${ }^{3}$ Ferguson GT, Enright PL, Buist AS et al. Office spirometry for lung health assessment in adults: A consensus statement from the National Lung Health Education Program. Chest 2000; 117: 1146-1161

${ }^{4}$ Halbert RJ, Isonaka S, George D et al. Interpreting COPD prevalence estimates: what is the true burden of disease? Chest 2003; 123: $1684-1692$

${ }^{5}$ Viegi G, Pedreschi M, Pistelli F et al. Prevalence of airways obstruction in a general population: European Respiratory Society vs American Thoracic Society definition. Chest 2000; 117: 339S-345S

${ }^{6}$ Schermer TR, Jacobs JE, Chavannes NH et al. Validity of spirometric testing in a general practice population of patients with chronic obstructive pulmonary disease (COPD). Thorax 2003; 58: 861 - 866

${ }^{7}$ Fletcher C, Peto R, Tinker C et al. The natural history of chronic bronchitis and emphysema. Oxford University Press, 1976

8 Pinto-Plata VM, Cote C, Cabral $\mathrm{H}$ et al. The 6-min walk distance: change over time and value as a predictor of survival in severe COPD. Eur Respir J 2004; 23: 28 - 33

${ }^{9}$ Nishimura K, Izumi T, Tsukino $\mathrm{M}$ et al. Dyspnea is a better predictor of 5 -year survival than airway obstruction in patients with COPD. Chest 2002; 121: $1434-1440$

10 O'Donnell DE, Lam M, Webb KA. Measurement of symptoms, lung hyperinflation, and endurance during exercise in chronic obstructive pulmonary disease. Am J Respir Crit Care Med 1998; 158: 1557- 1565

${ }^{11}$ O'Donnell DE. Assessment of bronchodilator efficacy in symptomatic COPD: is spirometry useful? Chest 2000; 117: 42S-47S

${ }^{12}$ O'Donnell DE, Revill SM, Webb KA. Dynamic hyperinflation and exercise intolerance in chronic obstructive pulmonary disease. Am J Respir Crit Care Med 2001; 164: 770-777

13 O'Donnell DE, Flüge T, Gerken F et al. Effects of Tiotropium on lung hyperinflation, dyspnoea and exercise tolerance in COPD. Eur Respir J 2004 ; in press

${ }^{14}$ Taube C, Lehnigk B, Paasch K et al. Factor analysis of changes in dyspnea and lung function parameters after bronchodilation in chronic obstructive pulmonary disease. Am J Respir Crit Care Med 2000; 162: $216-220$

15 Taube C, Kanniess F, Gronke L et al. Reproducibility of forced inspiratory and expiratory volumes after bronchodilation in patients with COPD or asthma. Respir Med 2003; 97: 568-577

${ }^{16}$ Saetta M, Turato G, Maestrelli P et al. Cellular and structural bases of chronic obstructive pulmonary disease. Am J Respir Crit Care Med 2001; 163: 1304-1309

${ }^{17}$ Turato G, Zuin R, Miniati M et al. Airway inflammation in severe chronic obstructive pulmonary disease: relationship with lung function and radiologic emphysema. Am J Respir Crit Care Med 2002; 166: $105-110$

${ }^{18}$ Retamales I, Elliott WM, Meshi B et al. Amplification of inflammation in emphysema and its association with latent adenoviral infection. Am J Respir Crit Care Med 2001; 164: 469-473

${ }^{19}$ Agusti A, MacNee W, Donaldson K et al. Hypothesis: Does COPD have an autoimmune component? Thorax 2003; 58: 832 - 834

${ }^{20} \mathrm{Holz}$ O, Zühlke I, Jaksztat $\mathrm{E}$ et al. Lung fibroblasts from patients with emphysema show a reduced proliferation rate in culture. Eur Respir J 2004; submitted

${ }^{21}$ Kips JC, Peleman RA, Pauwels RA. Methods of examining induced sputum: do differences matter? Eur Respir J 1998; 11: 529-533

${ }^{22}$ Tsoumakidou M, Tzanakis N, Siafakas NM. Induced sputum in the investigation of airway inflammation of COPD. Respir Med 2003; 97: $863-871$

${ }^{23}$ Taube C, Holz O, Mücke M et al. Airway Response to Inhaled Hypertonic Saline in Patients with Moderate to Severe Chronic Obstructive Pulmonary Disease. Am J Respir Crit Care Med 2001; 164: 1810- 1815

${ }^{24}$ Keatings VM, Barnes PJ. Granulocyte activation markers in induced sputum: comparison between chronic obstructive pulmonary disease, asthma, and normal subjects. Am J Respir Crit Care Med 1997; 155: 449-453

${ }^{25}$ Richter K, Holz O, Jorres RA et al. Sequentially induced sputum in patients with asthma or chronic obstructive pulmonary disease. Eur Respir J 1999; 14: 697 - 701

${ }^{26}$ Jayaram L, Parameswaran K, Sears MR et al. Induced sputum cell counts: their usefulness in clinical practice. Eur Respir J 2000; 16: $150-158$ 
${ }^{27}$ Hargreave FE, Leigh R. Induced sputum, eosinophilic bronchitis, and chronic obstructive pulmonary disease. Am J Respir Crit Care Med 1999; 160: S53-S57

${ }^{28}$ Kelly MM, Keatings V, Leigh R et al. Analysis of fluid-phase mediators. Eur Respir J Suppl 2002; 37: 24s-39s

${ }^{29}$ Kharitonov SA, Robbins RA, Yates D et al. Acute and chronic effects of cigarette smoking on exhaled nitric oxide. Am J Respir Crit Care Med 1995; 152: 609-612

${ }^{30}$ Repine JE, Bast A, Lankhorst I. Oxidative stress in chronic obstructive pulmonary disease. Oxidative Stress Study Group. Am J Respir Crit Care Med 1997; 156: $341-357$

${ }^{31}$ Hunt J. Exhaled breath condensate: an evolving tool for noninvasive evaluation of lung disease. J Allergy Clin Immunol 2002; 110: 28 - 34

${ }^{32}$ Schleiss MB, Holz O, Behnke $M$ et al. The concentration of hydrogen peroxide in exhaled air depends on expiratory flow rate. Eur Respir J 2000; 16: $1115-1118$

${ }^{33}$ Rahman I. Reproducibility of oxidative stress biomarkers in breath condensate: are they reliable? Eur Respir J 2004; 23: 183-184

${ }^{34}$ Magnussen $\mathrm{H}$, Richter K, Taube C. Are chronic obstructive pulmonary disease (COPD) and asthma different diseases? Clin Exp Allergy 1998; 28 Suppl 5: $187-194$

${ }^{35}$ Decramer M, de B V, Dom R. Functional and histologic picture of steroid-induced myopathy in chronic obstructive pulmonary disease. Am J Respir Crit Care Med 1996; 153: 1958 - 1964

${ }^{36}$ Singh JM, Palda VA, Stanbrook MB et al. Corticosteroid therapy for patients with acute exacerbations of chronic obstructive pulmonary disease: a systematic review. Arch Intern Med 2002; 162: 2527-2536

${ }^{37}$ Loppow D, Schleiss MB, Kanniess F et al. In patients with chronic bronchitis a four week trial with inhaled steroids does not attenuate airway inflammation. Respir Med 2001; 95: 115-121

${ }^{38}$ Culpitt SV, Maziak W, Loukidis S et al. Effect of high dose inhaled steroid on cells, cytokines, and proteases in induced sputum in chronic obstructive pulmonary disease. Am J Respir Crit Care Med 1999; 160: $1635-1639$

${ }^{39}$ Niewoehner DE, Erbland ML, Deupree RH et al. Effect of systemic glucocorticoids on exacerbations of chronic obstructive pulmonary disease. Department of Veterans Affairs Cooperative Study Group. N Engl J Med 1999; 340: 1941 - 1947

${ }^{40}$ Sutherland ER, Allmers H, Ayas NT et al. Inhaled corticosteroids reduce the progression of airflow limitation in chronic obstructive pulmonary disease: a meta-analysis. Thorax 2003; 58: 937 - 941

${ }^{41}$ Highland KB, Strange C, Heffner JE. Long-term effects of inhaled corticosteroids on FEV1 in patients with chronic obstructive pulmonary disease. A meta-analysis. Ann Intern Med 2003; 138: 969-973

42 Sin DD, McAlister FA, Man SF et al. Contemporary management of chronic obstructive pulmonary disease: scientific review. JAMA 2003; 290: $2301-2312$

${ }^{43}$ Sin DD, Man SF. Inhaled corticosteroids and survival in chronic obstructive pulmonary disease: does the dose matter? Eur Respir J 2003; 21: $260-266$

${ }^{44}$ Fan VS, Bryson CL, Curtis JR et al. Inhaled corticosteroids in chronic obstructive pulmonary disease and risk of death and hospitalization: time-dependent analysis. Am J Respir Crit Care Med 2003; 168: $1488-1494$

${ }^{45}$ Suissa S. Effectiveness of inhaled corticosteroids in chronic obstructive pulmonary disease: immortal time bias in observational studies. Am J Respir Crit Care Med 2003; 168: 49-53

${ }^{46}$ Rabe KF. Combination therapy for chronic obstructive pulmonary disease: one size fits all? Eur Respir J 2003; 22: 874 - 875

${ }^{47}$ Nannini L, Lasserson T, Poole P. Combined corticosteroid and longacting beta-agonist in one inhaler for chronic obstructive pulmonary disease. Cochrane Database Syst Rev 2003; 4: CD003794

${ }^{48}$ Calverley PM, Burge PS, Spencer S et al. Bronchodilator reversibility testing in chronic obstructive pulmonary disease. Thorax 2003; 58 : $659-664$

${ }^{49}$ Tashkin DP, Cooper CB. The role of long-acting bronchodilators in the management of stable COPD. Chest 2004; 125: 249-259

\section{Information}

\section{Informationsbericht \\ des DZK zur Bekämpfung der Tuberkulose}

Das Deutsche Zentralkomitee zur Bekämpfung der Tuberkulose (DZK) hat seinen 28. Informationsbericht veröffentlicht. Er enthält umfangreiche aktauelle Beiträge zur Internationalen und deutschen Tuberkuloseepidemiologie (inklusive Ergebnissen der DZK-Studien zur Epidemiologie in Deutschland), zu den Auswirkungen des Infektionsschutzgesetzes, eine Übersicht über die Aktivitäten und Publikationen des DZK und vieles mehr (Umfang 104 Seiten). Eine Zusammenstellung der epidemiologischen Tuberkulosestatistik liefert jetzt das Robert-Koch-Institut, da nach Infektionsschutzgesetz dieses nun für die Tuberkulose-Surveillance und Berichterstattung zuständig ist. Der DZK-Bericht stellt auch in seiner neuen Form für den Tuberkulose-Interessierten eine informative und interessante Lektüre dar. Er kann zum Preis von $€ 10,-$ zzgl. $7 \%$ Mwst. und Porto beim DZK bestellt werden.

Deutsches Zentralkomitee zur Bekämpfung der Tuberkulose Lungenklinik Heckeshorn

Zum Heckeshorn 33

14109 Berlin

Tel.: 030/8002 2435

Fax: 030/8002 2286

E-mail: loddheck@zedat.fu-berlin.de 